\title{
Staff versus Physician Vaccine Protocols for Influenza Immunization During Pregnancy
}

\author{
Leanne Zakrzewski, MD, Denise K. Sur, MD, and Nisha Agrawal, MD
}

Background: Pregnant women and their fetuses are known to be at increased risk for influenza-associated morbidity and mortality. The Advisory Committee on Immunization Practices and others have recommended influenza vaccination for all pregnant women at any gestational age, but vaccination rates for pregnant women remain low, near $45 \%$.

Methods: A retrospective chart review was performed at each of 4 sites during the 2010 to 2011 and 2011 to 2012 influenza seasons to examine the rates of flu vaccination offering and provision. The 4 separate clinics used for this study included 3 sites within a large university academic center and one private community practice. A nurse-driven (licensed vocational nurse or medical assistant staff) protocol for offering the flu vaccine was used at one site the first year of review and then used at 2 sites during the second year of review. We compared the vaccination offering and provision rates when nursedriven or physician-driven protocols were used.

Results: With the nurse-driven protocol, the average offering rate was $99.7 \%$, but of those who were offered the vaccine, only $38.2 \%$ received it, for a total effective vaccination rate of $38.1 \%$ of the patients. With the physician-driven protocol, $54.5 \%$ patients were offered the vaccine, and $70.7 \%$ of those received it, for an effective total vaccination rate of $38.5 \%$.

Conclusion: In this retrospective observational report, a nurse-driven protocol did not improve vaccination rates across varying practice sites. Thus, a simple protocol change to staff alone offering vaccine is unlikely to improve rates of maternal influenza vaccination. Additional studies looking at interventions to increase the number of pregnant women vaccinated against influenza are needed. $(\mathrm{J}$ Am Board Fam Med 2014;27:56-60.)

Keywords: Influenza, Pregnancy, Vaccination

Pregnant women and their fetuses are at increased risk for influenza-associated complications. Pregnant women infected with influenza have been shown to be at increased risk of hospitalization and admission to the intensive care unit; recent data from the Centers for Disease Control and Prevention (CDC) indicate that $65.9 \%$ of pregnant women with H1N1 were hospitalized in 2009 and

This article was externally peer reviewed.

Submitted 3 January 2013; revised 12 July 2013; accepted 22 July 2013.

From the Department of Family Medicine, University of California, Los Angeles, David Geffen School of Medicine, Santa Monica. (LZ).

Current affiliation: Kaiser Permanente, Los Angeles, CA

Funding: none.

Conflict of interest: none declared.

Corresponding author: Leanne Zakrzewski, MD, Kaiser Permanente, 12001 West Washington Boulevard, Los Angeles, CA 90066 (E-mail: leanne.m.zakrzewski@kp.org).
$24.7 \%$ of these women were admitted to the intensive care unit. ${ }^{1}$ One recent study showed a higher incidence of small-for-gestational age infants born to women who did not receive the influenza vaccine (44.8\% without vaccine vs $25.9 \%$ with vaccine; $P=$ $.03) .^{2}$ Influenza also affects many infants $<6$ months old, before they are eligible for vaccination, and can lead to hospitalization; therefore, maternal vaccination may protect infants against influenza via transplacental transfer of antibodies. ${ }^{3}$

Given this data, the Advisory Committee on Immunization Practices, the American Academy of Family Physicians, and the American College of Obstetricians and Gynecologists have recommended influenza vaccination for all pregnant women at any point during gestation. ${ }^{4,5}$ Despite this recommendation, many pregnant women still are not vaccinated. During the 2011 to 2012 season, using data from an Internet panel survey with 1660 
respondents conducted by the CDC, $47.0 \%$ of pregnant women received the influenza vaccine from any source, and only $43.7 \%$ of pregnant women were offered the vaccine by their health care providers. Women who received a recommendation from a health care provider were more likely to have been vaccinated than those who did not receive a recommendation $(81.6 \%$ vs $11.1 \%) .{ }^{6}$ The rate of maternal influenza vaccination noted above $(47.0 \%)$ is relatively consistent with the rate from the prior year, with $49.0 \%$ of pregnant women receiving the influenza vaccine in the 2010 to 2011 flu season. ${ }^{7}$

There are many possibilities and contributing factors explaining why health care providers are not offering influenza vaccination. A retrospective study at a university center in New Mexico examined multiple interventions to increase influenza vaccination rates among pregnant patients and found that standing orders, or a nurse-driven protocol, increased vaccination rates from $1 \%$ to $3 \%$ to $37 \%$ over 2 years. $^{8}$ Additional provider barriers to vaccinating pregnant patients include provider cost to stock and administer the vaccine and lack of provider knowledge of the current recommendation to vaccinate all pregnant women., ${ }^{9,10}$

On the basis of findings that women receiving a health care provider's recommendation were more likely to have been vaccinated against influenza, ${ }^{7,8}$ we conducted a study of an intervention that we hoped would increase the rate of influenza vaccinations being offered while simultaneously monitoring the percentage of women receiving the vaccine in the same population.

\section{Methods}

Four separate clinics were used for this study: 3 sites within a large university academic center and one private community practice, all within the West Los Angeles area. Of the university academic sites, 2 were residency clinics (the family medicine clinic and the obstetrics and gynecology [OB/ GYN] clinic), and the other was a private clinic staffed by university OB/GYN faculty physicians. The private community practice is not affiliated with the university academic center but is an OB/ GYN clinic located in the same West Los Angeles area. All 4 sites were selected based on variety of practice type and patient population. While the university $\mathrm{OB} / \mathrm{GYN}$ faculty practice and the pri- vate $\mathrm{OB} / \mathrm{GYN}$ clinic see primarily privately insured patients (an average of only $0.1 \%$ with Medicaid), both clinics staffed by residents see a much higher percentage of patients who are eligible for Medicaid. The OB/GYN residency clinic patient population is comprised $100 \%$ of patients who are eligible for Medicaid and the family medicine clinic patient population is comprised, on average, of $32 \%$ of patients who are eligible for Medicaid. The family medicine residency clinic includes a mixture of attendings and residents, whereas providers at the OB/GYN residency clinic were strictly residents. The OB/GYN faculty practice and the private $\mathrm{OB} / \mathrm{GYN}$ clinic physicians are all board-certified practicing physicians.

During the 2010 to 2011 influenza season, the academic faculty practice used a nurse-driven protocol for offering the flu vaccine; patients were offered and provided the vaccine by a licensed vocation nurse or medical assistant before the provider saw the patient. After the nurse administered a short questionnaire to ensure the patient did not have a known absolute contraindication to receiving the vaccine (including presence of fever and severe egg allergy, taken from the CDC guidelines) and provided a vaccine information sheet, the patient could receive her vaccine before the encounter with the provider. The nurse would then alert the physician or document in the chart the administration of the influenza vaccine. The physician would also be alerted if the patient declined the vaccine. The other 3 sites used a physician-driven protocol for offering the flu vaccine; the patient was offered the vaccine by the provider during the encounter. At the start of the 2011 to 2012 influenza season, the family medicine residency clinic changed to a nursedriven protocol but all other sites remained the same, bringing the total to 2 sites with a nursedriven protocol and 2 sites with a physician-driven protocol for the 2011 to 2012 influenza season.

A retrospective chart review was performed at each of the 4 sites at 2 points during the 2010 to 2011 and 2011 to 2012 influenza seasons. The first review was performed midway through the flu season, and the second review was performed 1 month after the end of the flu season. An administrator at each site developed a list of patients who were pregnant at any time during the influenza season. For our purposes, we defined the influenza season as when the vaccine was first available at each clinic to March 31. These lists were populated based on 
reviews of the database and appointments for patient visits during the influenza season at all sites except the family medicine residency site. There we reviewed the internal list of actively pregnant patients as well as the list of births at the affiliated hospital during the influenza season. We credited a physician for offering the flu vaccine if chart documentation indicated the patient was offered and/or given the vaccine during her pregnancy or during the postpartum period if she delivered in September or October. If there was no documentation, it was assumed that the patient was not offered the vaccine. There were no interruptions in the availability of the flu vaccine during either season or at any site throughout the study. We recorded the rates of influenza vaccination, as well as rates of providers offering the vaccine, at each site.

\section{Results}

At the family medicine residency clinic during the 2010 to 2011 season, 30 of 39 patients $(76.9 \%)$ were offered the vaccine, and $26(66.7 \%)$ received the vaccine. During the 2011 to 2012 season, when a nurse-based protocol was implemented, 47 of 54 patients $(87.0 \%)$ were offered the vaccine and 28 $(51.9 \%)$ received the vaccine. At the academic center physician practice, where a nurse-based protocol was in place for both seasons, all 822 patients were offered the vaccine (100\%), and 357 patients (43.4\%) received the vaccine during the 2010 to 2011 season. During the 2011 to 2012 season, all 1236 patients $(100 \%)$ were offered the vaccine, and 420 patients $(34.0 \%)$ received it.

At the $\mathrm{OB} / \mathrm{GYN}$ residency practice, the physician-driven protocol was used during both influenza seasons. During the 2010 to $2011 \mathrm{flu}$ season, 59 of 85 patients $(69.4 \%)$ were offered the vaccine, and 44 of them $(51.8 \%)$ received the vaccine. In the 2011 to 2012 season, 172 of 200 patients $(86.0 \%)$ were offered the vaccine and 100 patients $(50.0 \%)$ received it. At the private community $\mathrm{OB} / \mathrm{GYN}$ clinic, 30 of 209 patients (14.4\%) were offered the vaccine during the 2010 to 2011 influenza season, and $23(11 \%)$ received the vaccine. During the 2011 to 2012 season, 129 of 238 patients (54.2\%) were offered the vaccine, and 104 patients (43.7\%) received the vaccine.

When analyzing the effective vaccination rate between the 2 different protocols, the rates are very similar: $38.1 \%$ with the provider-driven protocol and $38.5 \%$ with the nurse-driven protocol. Vaccination recommendation and administration for each protocol are described in Table 1; Figure 1 shows the percentages of influenza vaccines offered and given at each site.

\section{Discussion}

Our study of 4 different practices over 2 influenza seasons found that a nurse-driven protocol was associated with an increased rate of offering vaccination compared with a physician-driven protocol, but no significant change in the effective vaccination rate was seen. While the rate of vaccine acceptance was higher when the nursing staff offered, the rate of patient immunization did not significantly change. This suggests possible barriers to vaccination of eligible women other than solely being offered the vaccine by any staff in the physician office. This is in line with the results of a recent meta-analysis highlighting the need for multiple types of interventions to increase influenza vaccination in older adults. ${ }^{11}$

There are a few factors that may have contributed to our findings. First, it is unclear whether a nursing staff member providing a recommendation for vaccination is perceived by the patient as equal to a physician's recommendation. Surveys of patient attitudes have evaluated this further and found multiple barriers, including safety concerns about ingredients, lack of knowledge about the influenza illness, and no history of receiving an earlier influenza vaccine, as reasons patients decline vaccination. ${ }^{12}$ More public outreach may be needed to change these perceptions of the vaccine and overall vaccination, as the physician alone often does not have sufficient time to discuss these concerns in

Table 1. Data Combining All Sites Over Both Influenza Seasons by the Provider- and Nurse-Driven Protocols

\begin{tabular}{|c|c|c|c|c|c|c|}
\hline & $\begin{array}{c}\text { Total } \\
\text { Patients (n) }\end{array}$ & Offered (n) & Given (n) & Offered (\%) & $\begin{array}{c}\text { Effective } \\
\text { Immunization (\%) }\end{array}$ & Acceptance (\%) \\
\hline Provider-driven protocol & 2112 & 2105 & 805 & 99.7 & 38.1 & 38.2 \\
\hline Nurse-driven protocol & 771 & 420 & 297 & 54.5 & 38.5 & 70.7 \\
\hline
\end{tabular}


Figure 1. Percentages of influenza vaccines offered and given at each site. 2010, 2010-2011 influenza season; 2011, 2011-2012 influenza season; FM res, family medicine residency practice; Nurse proc, nurse-driven protocol; $\mathrm{OB}$ fac: obstetrics faculty practice; $\mathrm{OB}$ res, obstetrics residency practice; Prov proc, provider-driven protocol; Prvt OB, private obstetrics community practice.
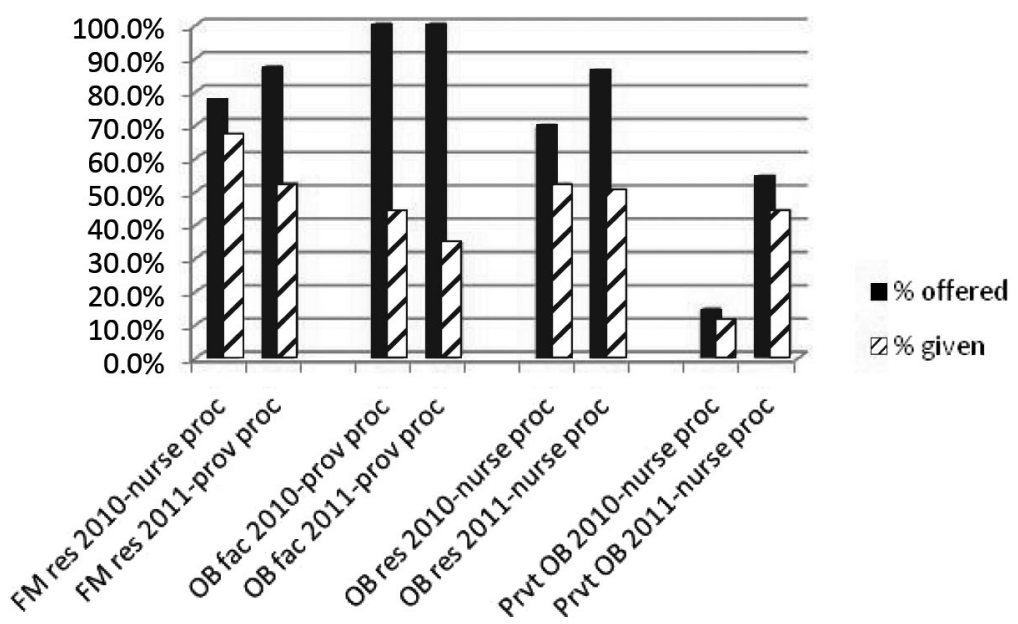

detail with each patient. Physician barriers to offering and providing the influenza vaccine also have been evaluated and indicate that financial constraints regarding reimbursement and costs of ordering and storing vaccines are significant barriers to obstetric physicians. ${ }^{10}$ In addition, many obstetric physicians feel their knowledge of and training on the usefulness and safety of vaccines is lacking, ${ }^{10}$ and so additional physician education classes are needed. Studies have shown physician education outreach can increase vaccination rates, ${ }^{13}$ and this is an area that can be further examined, including cost-benefit analyses of different outreach methods.

Regarding our research, it is notable that the vaccination rates during both influenza seasons were considerably higher than national averages at the family medicine residency site, but only a small number of patients were included at this site and so this could account for the higher averages. This low number may also limit our ability to demonstrate improved rates with the implementation of a nursedriven protocol. The higher rate could be persistent at higher patient numbers, however, and this may be secondary to the academic setting of this site, where regular continuing medical education classes are held and trainees are involved. Levels of influenza vaccination higher than the national average were not seen, however, in the OB/GYN residency practice, which involves trainees and where a separate continuing medical education curriculum is in place. This indicates that other fac- tors, as outlined earlier, likely play a significant role in patient receipt of the influenza vaccine.

Our research also indicates that overall vaccination rates went down at 3 of the 4 clinics (all except the private $\mathrm{OB} / \mathrm{GYN}$ practice) between the 2 influenza seasons. This could be related to the H1N1 pandemic of 2010, along with its associated media coverage and patient awareness of the influenza illness, which might have driven more patients to receive the influenza vaccine. Because such public discussion and awareness of influenza has lessened with each year since $\mathrm{H} 1 \mathrm{~N} 1$, vaccination rates may also be falling.

Finally, our data show that rates of offering and administering vaccinations did increase at the private $\mathrm{OB} / \mathrm{GYN}$ community practice over the two influenza seasons. We believe this is likely due to increasing physician awareness about the influenza vaccine by the presence of our research team performing chart reviews in the office during each flu season. This clinic already had the influenza vaccine in stock during the 2010 to $2011 \mathrm{flu}$ season, and the only change between the 2 seasons was our presence in their medical records office. The increase in influenza vaccine administration between the 2 seasons is likely secondary to the increase in physician recommendation and offerings, which parallels the results of other studies. ${ }^{8,13}$

Limitations of our study include the low numbers of patients from each individual site, as noted earlier, as well as our method of calculating rates of 
influenza vaccine offerings by physicians via chart documentation; a physician may have offered the vaccine but failed to document it in the patient's chart. Last, since our study was limited to the Los Angeles area, it is unclear what regional variations in attitudes toward vaccinations may have contributed to our findings.

\section{Conclusions}

The number of eligible pregnant patients who were offered the influenza vaccine increased from the 2010 to $2011 \mathrm{flu}$ season to the 2011 to $2012 \mathrm{flu}$ season at all sites studied. Higher rates of vaccination offerings were demonstrated at sites using nurse-driven protocols compared with physiciandriven protocols. An increase in the number of vaccine offerings unfortunately did not result in an increase in the effective vaccination rate. Additional studies looking at interventions to increase the number of pregnant women vaccinated against influenza are needed.

The authors thank Drs. Alana Ugell Franklin, Sandra Vizireanu, and Mona Cho for their research assistance, as well as physicians and staff at each study site for their time and cooperation.

\section{References}

1. Siston AM, Rasmussen SA, Honein MA, et al; Pandemic H1N1 Influenza in Pregnancy Working Group. Pandemic 2009 influenza A (H1N1) virus illness among pregnant women in the United States. JAMA 2010;303:1517-25.

2. Steinhoff MC, Omer SB, Roy E, et al. Neonatal outcomes after influenza immunization during pregnancy: a randomized controlled trial. CMAJ 2012; 184:645-53.

3. Poehling KA, Edwards KM, Weinberg GA, et al. The underrecognized burden of influenza in young children. N Engl J Med 2006;355:31-40.
4. Fiore AE, Uyeki TM, Broder K, et al.; Centers for Disease Control and Prevention. Prevention and control of influenza with vaccines: recommendations of the Advisory Committee on Immunization Practices (ACIP), 2010. MMWR Recomm Rep 2010; 59(RR-8):1-62.

5. American Congress of Obstetricians and Gynocologists Committee on Obstetric Practice. ACOG committee opinion no. 468: influenza vaccination during pregnancy. Obstet Gynecol 2010;116:1006-7.

6. Centers for Disease Control and Prevention (CDC). Influenza vaccination coverage among pregnant women: 2011-2012 influenza season, United States. MMWR Morb Mortal Wkly Rep 2012;61:758-63.

7. Centers for Disease Control and Prevention (CDC). Influenza vaccination coverage among pregnant women-United States, 2010-11 influenza season. MMWR Morb Mortal Wkly Rep 2012;60:1078-82.

8. Ogburn T, Espey EL, Contreras V, Arroyo P. Impact of clinic interventions on the rate of influenza vaccination in pregnant women. J Reprod Med 2007; 52:753-6.

9. Kissin DM, Power ML, Kahn EB, et al. Attitudes and practices of obstetrician-gynecologists regarding influenza vaccination in pregnancy. Obstet Gynecol 2011;118:1074-80.

10. Power M, Leddy M, Anderson B, et al. Obstetriciangynecologists' practices and perceived knowledge regarding immunization. Am J Prev Med 2009;37: 231-4.

11. Lau DL, Hu J, Majumdar SR, et al. Interventions to improve influenza and pneumococcal vaccination rates among community-dwelling adults: a systematic review and meta-analysis. Ann Fam Med 2012; 10:538-46.

12. Shavell VI, Moniz MH, Gonik B, Beigi RH. Influenza immunization in pregnancy: overcoming patient and health care provider barriers. Am J Obstet Gynecol 2012;207(3 suppl):S67-74.

13. Panda B, Stiller R, Panda A. Influenza vaccination during pregnancy and factors for lacking compliance with current CDC guidelines. J Matern Fetal Neonatal Med 2011;24:402-6. 